\title{
International Group Work for Sustainable Chemistry
}

\author{
Katherine J. Haxton* and Richard J. Darton \\ School of Chemical and Physical Sciences, Keele University, Keele \\ *Corresponding author: k.j.haxton@keele.ac.uk
}

Keywords: Internationalisation; Sustainable Development; Group Work; Transnational Education

\begin{abstract}
As part of a transnational degree programme, students in the UK and China were linked together to carry out group work. This was enabled by having two versions of our FHEQ Level 4 Sustainable Chemistry module; in person for UK students, and by distance learning for Chinese students. Groups were instructed to discuss issues relating to the UN Sustainable Development Goals in their respective countries and report back individually. Students who found the assignment interesting were more likely to indicate they would keep in touch, while those students who had fewer interactions or found the assessment guidelines unclear found the assignment less interesting and were less likely to wish to remain in touch. This kind of assignment is an example of what can be done with links to other institutions around the world.
\end{abstract}

\section{Introduction}

There have been significant driving forces towards better integration of Education for Sustainable Development (ESD) in English Higher Education in the past decade. These include the United Nations Decade of ESD between 2005 - 2014, (Buckler \& Creech, 2014), the Higher Education Academy (2012) and the National Union of Students (2018). These, combined with the expectations of staff and students that sustainable development will be incorporated into degree programmes, have led to an increase in visibility of the area. While ESD has the potential to be transformative, it is recognised that sometimes it is necessary to make incremental changes (Bessant et al., 2015). Our module on Sustainable Chemistry was introduced at Keele University (KU) in 2013 initially at FHEQ level 5 and has since been expanded and adapted for level 4 inperson and distance learning formats. The heart of Sustainable Chemistry is placing chemistry within the societal, political, economic, financial and environmental contexts, also framed as systems thinking (Matlin et al., 2016). Through sessions on food and water security, energy, plastics, personal and health care, and future developments in chemistry we challenge students to consider the broader implications of chemistry on society and the planet. We go beyond Green Chemistry (Anastas \& Kirchhoff, 2002) and consider the role of chemistry in making progress towards the UN Sustainable Development Goals (United Nations, 2015).

\section{Transnational Degree Programmes}

Transnational degree programmes are becoming increasingly common in Higher Education in England, and globally. Transnational education is defined as "any teaching or learning activity in which students are located in different countries to that in which the institutions providing the education is based". (Global Alliance for Transnational Education 1997 definition as cited in Alaba, 2014). Typically, students will be taught by the English institution in their country of origin, and be taught at the English university. Many programmes that require completing the degree in England cover the essential content 


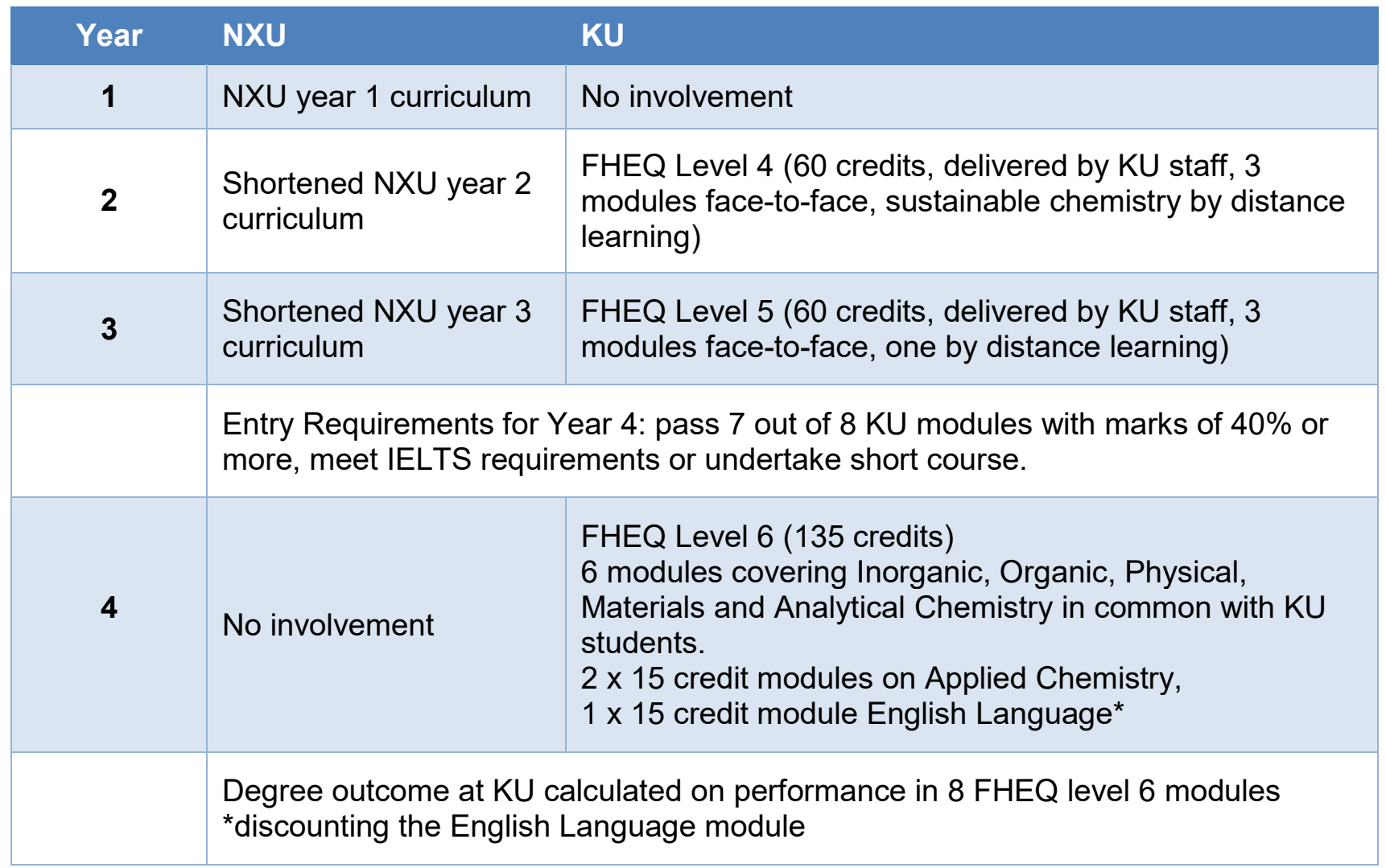

Table 1 Structure of BSc Applied Chemistry transnational degree between KU and NXU.

for that part of the programme through modules delivered by distance learning or by teaching staff travelling to the international institution (Robinson et al. 2016).

Typically, students will be taught by the English institution in their country of origin, and be taught at the English university. Many programmes that require completing the degree in England cover the essential content for that part of the programme through modules delivered by distance learning or by teaching staff travelling to the international institution (Robinson et al. 2016).

There are additional challenges for the students arriving in England who must integrate with an existing cohort of students and study at higher levels (FHEQ level 5 - 7) and may be faced with obtaining a degree classification based on one year of study in England.

In $2014 \mathrm{KU}$ established a collaborative degree programme with Nanjing XiaoZhuang University (NXU). This involved a combination of study at NXU in English and Chinese, a series of bridging modules at FHEQ level 4 and 5 delivered by $\mathrm{KU}$ staff, then completion of FHEQ level 6 at KU. The bridging modules were designed to provide chemical knowledge, experience of teaching styles and methods used in England, and assessment in English linked to entry requirements for the final year at $\mathrm{KU}$. Students on this programme work towards BSc Applied Chemistry and join the KU BSc Chemistry students in the final year (FHEQ level 6). Students studying the BSc Applied Chemistry course at NXU must pass 7 out of 8 bridging modules during years 2 and 3 at NXU and the award of BSc Applied Chemistry is only based on the 120 credits at FHEQ level 6, discounting an English Language support module (table 1).

\section{Sustainable Chemistry}

Our Sustainable Chemistry module was ideal for adapting to distance learning as part of the bridging module curriculum. From a teaching perspective, adapting the course to assume less cultural knowledge of England and Western countries was fascinating and 
challenging. Inherent within ESD is the idea that our students need to start thinking globally and international group work provides an ideal opportunity for students in England and China to learn from one another and to look for the differences and similarities to their approach towards sustainable development issues. By synchronising the modules, we provided an opportunity for students in the UK and China to interact and learn from one another, using email or social media. A short report was then produced individually by students in both cohorts documenting and reflecting on their learning. This task also allowed the two cohorts to interact in advance of arrival. Various schemes have been reported to facilitate interaction between students on collaborative transnational degree programmes pre-arrival, for example introductory videos (Sedghi UKCISA; Willis \& Sedghi 2014) and multicultural group work on after arrival (Sedghi \& Rushworth 2017). This is viewed as useful in mitigating culture shock for the incoming students and creating a welcoming and supportive atmosphere.

\section{Structure}

The distance-learning module took place over the full academic year (October - May) while the in-person version was in the $2^{\text {nd }}$ semester (January - May). Additionally, the timetable had to accommodate examination periods, Christmas and New Year in the UK, and the Lunar New Year in China. One of the short assignments in each module was designed as international group work. In this way it was low risk but with sufficient marks to inspire engagement. The distance-learning module was split into two sections before and after holidays in January/February. In the second section, and to align with the $\mathrm{KU}$ module, the topics were covered in the same weeks in both courses.

\section{Goals of the assignment:}

- To learn about sustainability in another culture (UK or China)

- To interact with students in China or the UK and learn about their culture

- To build interactions between KU and NXU students prior to $3^{\text {rd }}$ year when the cohorts will share classes.
In this study we report the student evaluation of international group work, and how engagement with this task relates to students' desire to remain in touch with each other, and consider some of the practical and ethical issues around international group work.

\section{Evaluation}

Evaluation of the activity was carried out via the Google form for submission by the KU students and as part of the module evaluation delivered by a colleague in-person at NXU. On the advice of colleagues, the evaluation questions for NXU were phrased as direct questions rather than statements. Our main goal in the evaluation was to establish what topics were discussed (self-reported by the KU students) and whether the students intended to remain in contact.

Evaluation questions for KU students (all Likert style) were:

- I found this assignment interesting

- I would have preferred to carry out independent research to complete this assignment.

- Most of my group responded to emails

- I will keep in touch with Chinese students from my group.

- A discussion forum or blog would have been better for this assignment.

- I found the assignment guidelines clear.

Evaluation questions for NXU students were:

- Was emailing the Keele students interesting? [Yes/Maybe/No]

- Will you keep in contact with some of the Keele students? [Likert]

- How could we make this task better? [Free Text]

\section{Ethical Considerations and Data Analysis}

As this was evaluation of a teaching activity, ethical approval was not sought. The results are presented here under service level evaluation of a teaching activity. We wanted to gauge the intent to remain in touch to facilitate further pre-arrival interaction between the cohorts. Optional survey responses were removed from the $\mathrm{KU}$ GoogleForm before marking for the assessment elements. The 


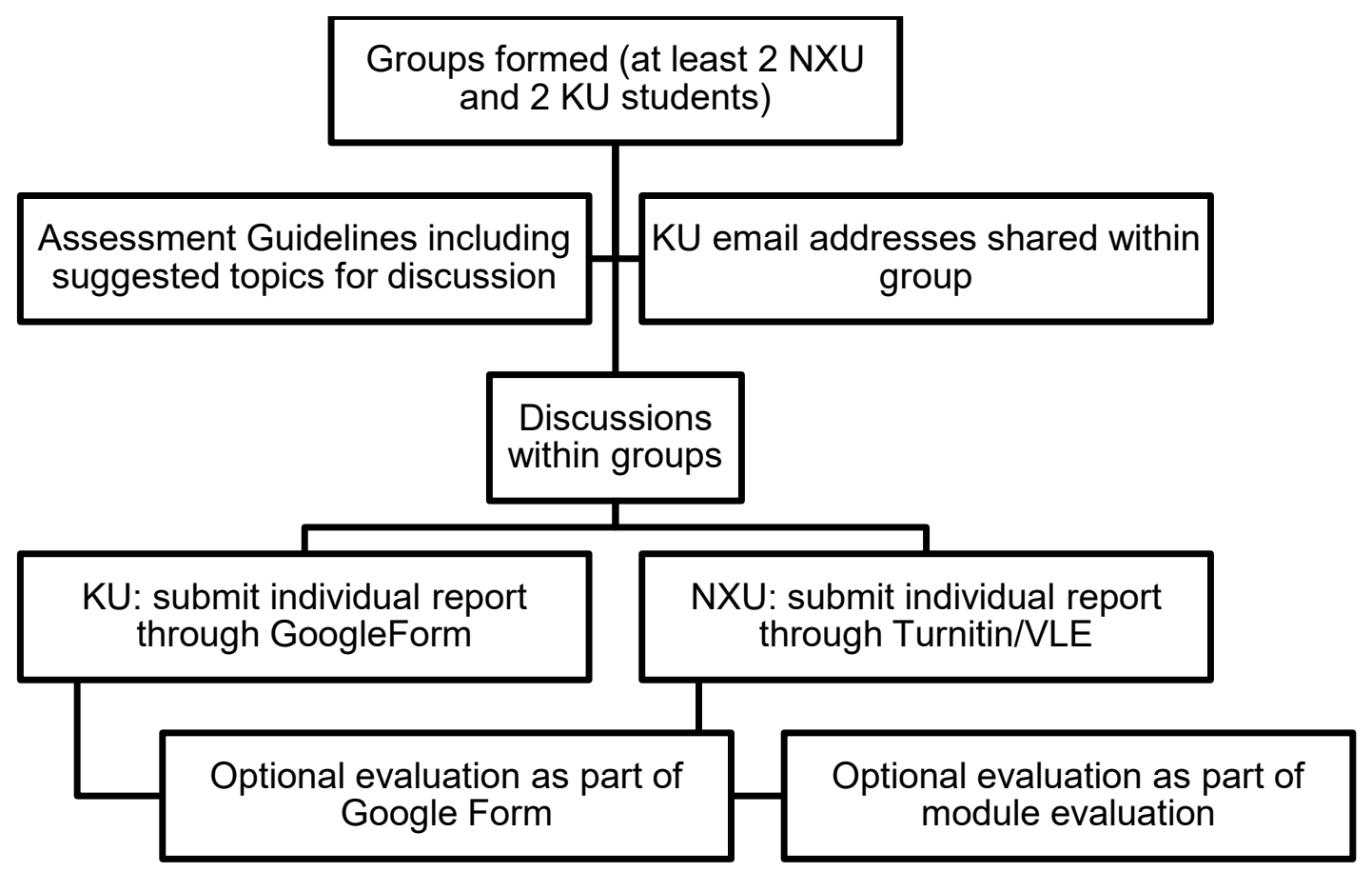

Figure 1 Structure of assignment and evaluation.

\begin{tabular}{|c|c|c|c|}
\hline Cohort & KU & NXU & No. of groups \\
\hline $\mathbf{1}$ & 59 & 37 & 18 \\
\hline $\mathbf{2}$ & 68 & 34 & 17 \\
\hline
\end{tabular}

Table 2 Cohort sizes, numbers of groups

survey responses were anonymised. NXU students completed a written module evaluation form delivered face-to-face at NXU. Hand written responses were transcribed by a colleague into Excel and were anonymous. Analysis was performed using Excel and the StatsPlus plugin, using Norman (2010) to justify statistics using Likert type data. No quotations from free text responses are reported as no permission to use quotations was sought.

Giving out students' email addresses to facilitate contact was given careful consideration. We also did not give out names and recommended that the first email be a personal introduction to start the conversation. This also allowed students at the same institution to introduce themselves to each other. We were aware of various cultural conventions that may have caused confusion for many students, such as the different format for Chinese names (family name, given name) and English names (given name, family name), and the preference of some Chinese students to use English names.

The form for submission of work at KU allowed the opportunity for additional questions to be asked around the logistics of the assignment. The questions were retained in both years but the data from cohort 1 was used as a preliminary evaluation to enhance the assessment guidelines. Our reflection on general feedback from NXU students on IT issues led to enhancements to the assessment guidelines.

\section{Practicalities}

This international group work assignment was worth $10 \%$ of a 15 -credit module and the structure is outlined in figure 1 . In creating the groups, no consideration was given to the gender of the students. The task was introduced several weeks before the deadline. Students were warned about the time difference and that response times to messages would vary. Problems caused by a 


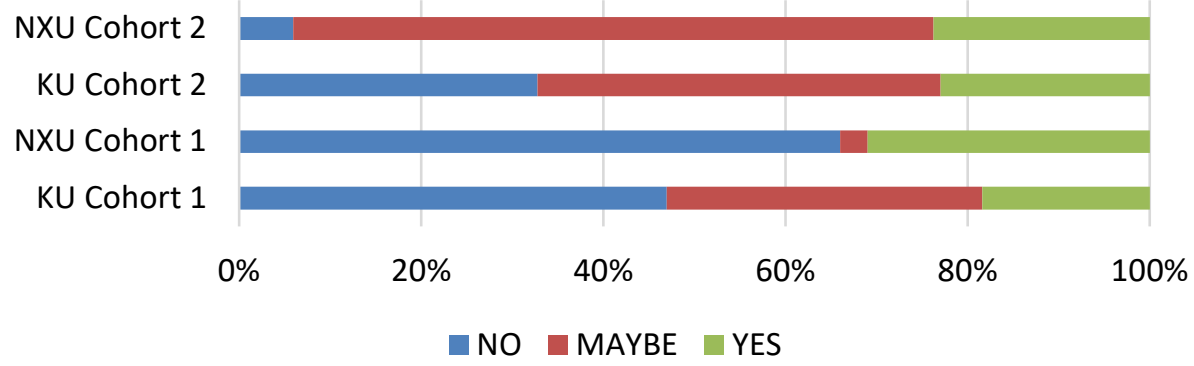

Figure 2 NXU: Will you keep in contact with some of the KU students? (Yes, No, Maybe) $\mathrm{KU}$ : I will keep in touch with Chinese students from my group (strongly agree/agree merged into 'Yes', neutral into 'Maybe' and disagree/strongly disagree into 'No').

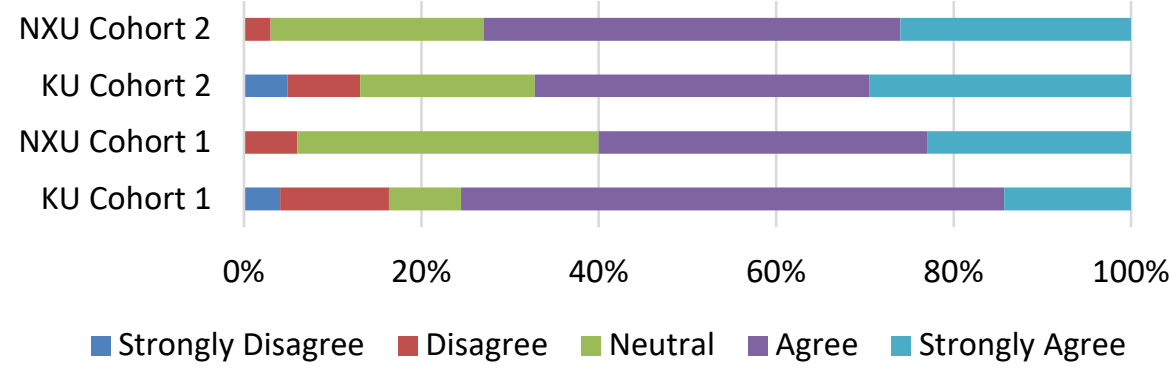

Figure $3 \mathrm{NXU}$ : Was emailing the KU students interesting? KU: I found this assignment interesting. Due to an error in the questionnaire the NXU version was left as a Likert style scale rather than a direct question.

lack of response was noted in feedback from both Chinese and UK students and may well reflect the diversity of attitudes within each cohort towards group work, the task or organisation. Several students did not engage with the task at all and the reasons for this were not probed further. In year 1 , all students were asked only to use their KU email accounts which created additional issues. Due to issues with internet access in China, students are forced to use a virtual private network to access KU servers. This is often restricted and so NXU students had limited opportunity to access the VLE, their KU email account and communicate with their groups. It was surprising how few groups overcame this barrier - some groups signed up for WeChat accounts (a phone based messenger app) and carried out extensive discussions that way. In year 2 we recommended WeChat and suggested that the NXU students could use their personal email accounts if they wanted which facilitated far greater interaction. The severity of the internet access issues varied from student to student at NXU. No internet access issues were reported by KU students.
When considering the possible technologies for this assignment, discussion forums and blogs through the VLE were investigated, however accessing the VLE was very challenging for students in China and so email was identified as the best technology.

Assessment guidelines were modified between the two cohorts which had some positive effect on the students' perceptions of their clarity. Fewer emails asking for clarification were received from cohort 2 .

\section{Results}

The key aspects of the evaluation for both $\mathrm{KU}$ and NXU students revolved around whether the students intended to remain in touch, and whether the assignment was interesting. Between cohorts 1 and 2, there was a general shift towards keeping in touch in some format (figure 2).

This is most likely because communication was generally easier between the groups once we removed the constraint of communicating only via $\mathrm{KU}$ email accounts. Interest in the assignment followed a similar trend to keeping in touch (figure 3). The majority of students 


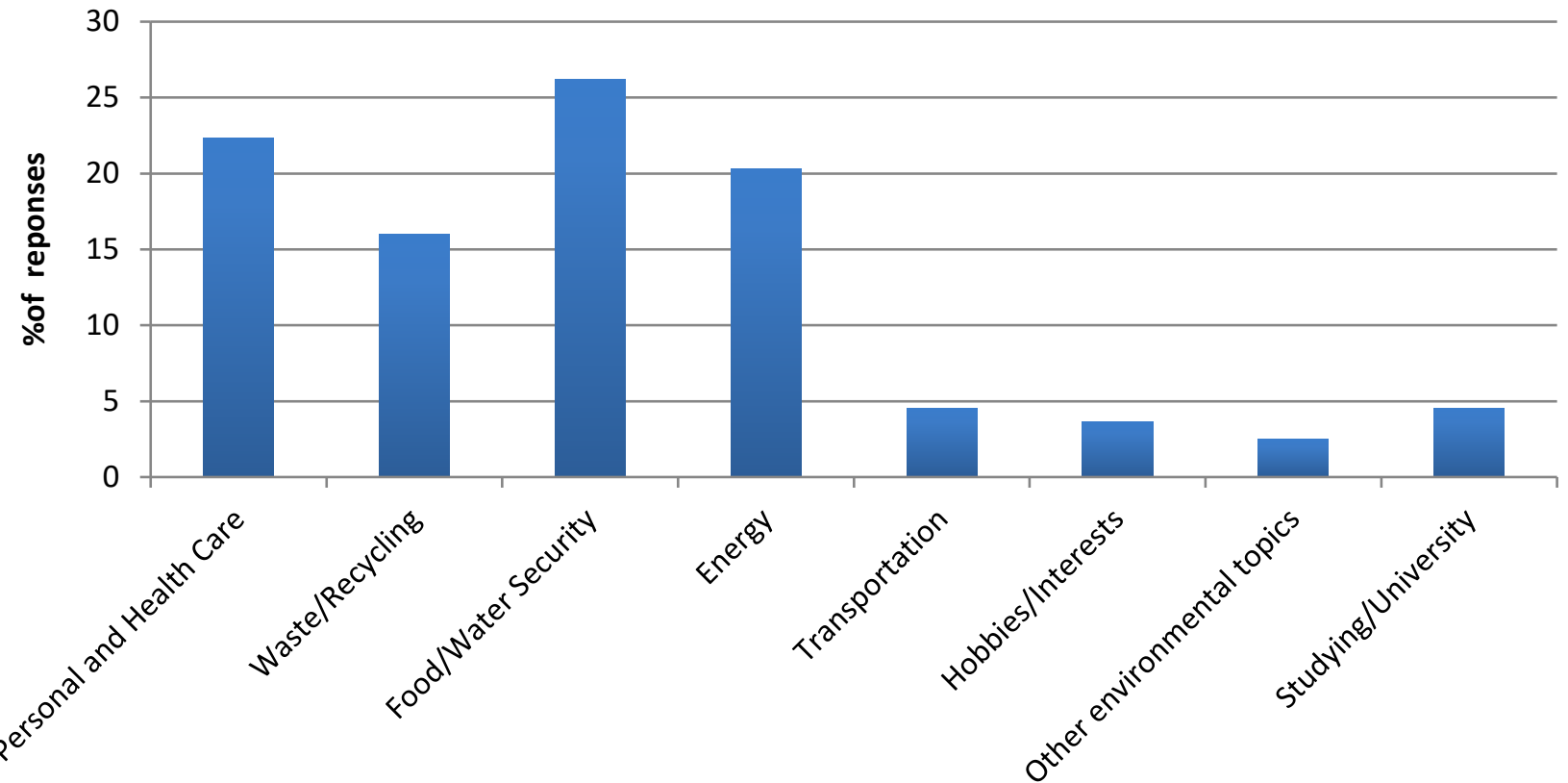

Figure 4 Thematically grouped topics of discussion for both years, derived from selfreporting of discussion topics by KU students.

found the assignment interesting, with a greater proportion in cohort 2 noting agreement or strong agreement.

Further analysis of the $\mathrm{KU}$ evaluation data indicates students may be split into two main groups: those that felt generally positively about the task and had some intention to remain in contact, and those who felt negatively about the task.

Interest in the assignment was the key predictor as to whether the KU students intended to remain in touch with the NXU students $(r=0.644, \quad n=110, \quad p<0.001)$. Interest also correlated positively with ease of communications $(r=0.288, \quad n=110, \quad p<$ 0.001 ), perception of clarity of assessment guidelines $(r=0.335, \quad n=110, \quad p<0.001)$. The degree of communication between group members (KU: most of my group responded to emails) also correlated positively with an intention to keep in touch $(r=0.288, n=110$, $p<0.01)$. KU students with a strong preference for carrying out independent research were less interested in the task $(r=$ $-0.56, n=110, p<0.001$ ), lack of response to emails $(r=-0.327, n=110, p<0.0001)$ and intention to keep in touch $(r=-0.5270, n=$ $110, p<0.0001)$.
The topics of discussion reported by $\mathrm{KU}$ students generally related to the topics taught in the module (figure 4 ) and over $80 \%$ related to the UN Sustainable Development goals. Unsurprisingly the conversations evolved to cover hobbies and interests, and the natural common ground: Keele University and staff teaching. Many of the conversations related to sustainability issues in practice such as transport - use of public transport and ease of use. From the written reports, it was clear that both sides uncovered many differences and similarities between England and China.

\section{Discussion}

Asking two distinctive groups of students to interact and then complete an assessed item of coursework has many risks and many rewards. From both sides, there is some familiarity with the other's culture, which may obscure key differences and lead to incidents when navigating social and personal boundaries in engagements (Hoare 2013). In suggesting topics for this assignment, consideration was given to the level of personal information that students would feel comfortable disclosing and whether that would vary between cultures. This can lead to intercultural transgressions (Pyvis \& Chapman 2005; Hoare 2013), which may be detrimental to the students' interactions and then success 
in the assignment. This was mitigated by facilitating interactions within groups rather than 1 on 1 and encouraging group emails or chat via social media apps. The topics suggested related to the UN Sustainable Development Goals and the taught content in the course to provide common ground and assist in overcoming this.

The extent of the challenge caused by internet access for the NXU students was not anticipated. The internet access issues also impacted on other aspects of distance learning including the ease of viewing lecture recordings and screencasts, participation in forums and on occasion, submission of work. Advising students to make initial contact through KU email accounts and then consider WeChat was effective, as was permitting use of personal emails if the students were comfortable doing so.

Clear positive correlations between interest in the assignment and email responses, keeping in touch beyond the assignments and the clarity of the assessment guidelines are noted. In general, students who indicated a strong preference for independent research as an alternative assignment found the task less interesting, were more likely to note a poor response to emails, and were less likely to indicate they wished to keep in touch. Some of these aspects could be improved on if this task were to be run again: groups could be made larger to increase the chances of positive interactions, more structured interactions could be implemented to help students overcome any hesitation in engaging. Independent research to supplement the information from the discussions, or as an alternative assessment allowing the student some choice. We went into this assignment with no preconceived notions of what should be discussed beyond issuing a series of prompts based on the module sessions. The aim was to give the students freedom to allow their interactions to happen without too many constraints. Many unexpected topics of conversation arose, particularly around the common ground for these students: the KU teaching staff and course content/structure. Where the students were engaged with the tasks, the conversations were rich and fruitful and went far beyond the scope of the course.

\section{Conclusions}

Group work of this sort provides a valuable opportunity for students to interact and learn through asynchronous dialogue. This was facilitated by having a module that could be run as both face-to-face $(\mathrm{KU})$ and distance learning (NXU) versions. This gave the students common ground and provided a framework on which to base their discussions. Over $80 \%$ of discussion was based on the UN Sustainable Development goals. Further evaluation is needed to investigate whether this had a longer-term impact on the students remaining in touch before and after arrival at KU. Assessment guidelines and weighting should be sufficient to inspire confidence in the students and compel them to engage well with the task, but not so high that slow or no responses are a source of significant additional pressure or stress. While discussion forums may initially seem ideal for this type of interaction, very careful consideration must be given to the accessibility of the forums based on local internet access. It is not necessary for staff to have oversight of the communications. Freedom to select the most appropriate methods of communication within a group inspires better interaction, which in turn leads to greater intent to remain in touch.

\section{Acknowledgements}

Dr Daniela Plana for administering and transcribing the NXU evaluation questionnaires.

\section{References}

Alaba, S.O. (2014) Information and Communication Technologies as a Tool for Enhancing Meaningful and Sustainable Transnational Collaboration in Education. Mediterranean Journal of Social Sciences 5 (23): 1465-1469 DOI: 10.5901/mjss.2014.v5n23p1465

Anastas, P.T. \& Kirchhoff, M.M. (2002) Origins, Current Status, and Future Challenges of Green Chemistry. Accounts of Chemical Research 35 (9). DOI: 10.1021/ar010065m

Bessant, E.F., Robinson, Z.P. \& Ormerod, R.M., (2015) Neoliberalism, new public management and the sustainable development agenda of higher education: history, contradictions and synergies. Environmental 
Education Research. 21(3): 417-432. DOI: 10.1080/13504622.2014.993933

Buckler C. \& Creech, H. (2014) Shaping The Future We Want: UN Decade of Education for Sustainable Development Final Report. Available at: http://unesdoc.unesco.org/images/0023/0023 01/230171e.pdf [Accessed March 2018]

Higher Education Academy (2012) Education for sustainable development in higher education. Available at: https://www.heacademy.ac.uk/individuals/strat egic-priorities/education-sustainabledevelopment [Accessed August 2018]

Hoare, L. (2013) Swimming in the deep end: transnational teaching as culture learning? Higher Education Research \& Development, 32(4): 561-574, DOI: $10.1080 / 07294360.2012 .700918$

Matlin, S., Mehta, G., Hopf, H. \& Krief, A. (2016) One-world chemistry and systems thinking. Nature Chemistry, 8(5), 393-398. DOI: 10.1038/nchem.2498

National Union of Students (2018) Sustainability. Available at: https://sustainability.nus.org.uk/ [Accessed August 2018]

Norman, G. (2010) Likert scales, levels of measurement and the "laws" of statistics. Advances in Health Science Education, 15, 625 - 632. DOI: 10.1007/s10459-010-9222-y

Pyvis, D. \& Chapman, A. (2005). Culture shock and the international student 'offshore'. Journal of Research in International Education, 4(1), 23-42. DOI: 10.1177/1475240905050289
Robinson, Z.P., Cage, A.G., Szkornik, K., Haffenden, A., Oliver, I., Stimpson, I., Stott K., Ullah S. \& Waller R.I. (2016). The Rise and Fall of a Collaboration: Reflections on the benefits, challenges and lessons learned from a joint England-China teaching initiative. The Journal of Academic Development and Education, 3256. Available at: https://jadekeele.files.wordpress.com/2013/04 liade-international-edition.pdf

Sedghi, G. (2013) Developing strategies and activities to improve interactions between home and international students and to enhance teaching and learning. UKCISA/HEA Available at: https://www.heacademy.ac.uk/system/files/liv erpool sedghi connections final report.pdf [Accessed March 2018]

Sedghi, G. \& Rushworth, E. (2017) The relation between multi-cultural group work and the integration of home and international students. New Directions in the Teaching of Physical Sciences, Volume 12, Issue 1. DOI: 10.29311/ndtps.v0i12.857

United Nations (2015) Sustainable Development Goals. Available at: http://www.un.org/sustainabledevelopment/su stainable-development-goals/ [Accessed March 2018]

Willis, I. \& Sedghi, G. (2014) Perceptions and experiences of home students involved in welcoming and supporting direct entry 2nd year international students. Practice and Evidence of Scholarship of Teaching and Learning in Higher Education 9 (1), 2-17 http://community.dur.ac.uk/pestlhe.learning/in dex.php/pestlhe/article/view/96 